\title{
Tunneling Effect in Quantum Hall Device*
}

\author{
Shosuke Sasaki ${ }^{\dagger}$ \\ Shizuoka Institute of Science and Technology, Toyosawa 2200-2, Fukuroi, Shizuoka 437-8555, Japan
}

(Received 3 September 2009; Accepted 4 February 2010; Published 13 March 2010)

\begin{abstract}
In usual quantum Hall devices, electric current flows through a thin layer with a uniform thickness. We consider a new quantum Hall device which has an potential barrier in the layer. Then the electrons flow by a tunnelling effect through the potential barrier. We investigate phenomena on the new quantum Hall device. Then voltage steps may appear in the curve of voltage $V$ versus electric current $I$, when the magnetic field value is modulated by oscillation with a constant frequency $f$. The step value $V$ is related to the transfer charge $Q$ as $V=(2 \pi \hbar f) /|Q|$. The value of the transfer charge $Q$ depends on the quantum Hall state. The usual theory implies that this value $|Q|$ is $e$ (elementary charge) for an integer quantum Hall state, and is $\nu e$ for a fractional quantum Hall state with fractional filling factor $\nu$. We examine the structure of the devices and show three types of them. Thereafter observation of this tunneling effect is investigated in details and it is clarified that the frequency value $f$ should be within the special region. We find that the curve of $(I, V)$ has several shapes.
\end{abstract}

[DOI: $10.1380 /$ ejssnt.2010.121]

Keywords: Many body and quasi-particle theories; Hall effect; Semiconductor-semiconductor heterostructures; Nanoelectronics and related devices

\section{INTRODUCTION}

The value of diagonal resistance in integer quantum Hall effect (IQHE) is almost zero [1-3]. This property indicates that the electron scatterings via thermal motion are extremely small in the integer quantum Hall states (IQH states). For the fractional quantum Hall states (FQH states) with filling factors $1 / 3,2 / 3$ etc., the diagonal resistance is also almost zero [3].

These properties make it possible to observe a tunneling effect in a quantum Hall device, if new experiments are carried out using new devices where potential barrier is put in the electron channel. The length of the potential barrier needs to be smaller than about $2 \mathrm{~nm}$ for quantum transition. Accordingly, we can consider three types of new devices as follows;

- (Type 1) A narrow potential barrier is put in an electron channel of a Hall device as illustrated in Fig. 1. The length of the barrier should be smaller than $2 \mathrm{~nm}$. Accordingly, it is slightly difficult to make this type of device.

- (Type 2) Other types of quantum Hall devices are illustrated in Fig. 2. We show two types of new devices with single gate or three gates. We can create an appropriate potential barrier from adjusting the voltage of the central gate in Fig. 2. It is easier to make the type 2 of the device than type 1.

- (Type 3) The central part of electron channel has a thickness less than one in the other part. This structure is illustrated in the side view of Fig. 3, where the $2 \mathrm{D}$ electron system is connected through

\footnotetext{
*This paper was presented at 10th International Conference on Atomically Controlled Surfaces, Interfaces and Nanostructures (ACSIN-10), Granada Conference Centre, Spain, 21-25 September, 2009 .

$\dagger$ Corresponding author: sasaki@ns.sist.ac.jp
}

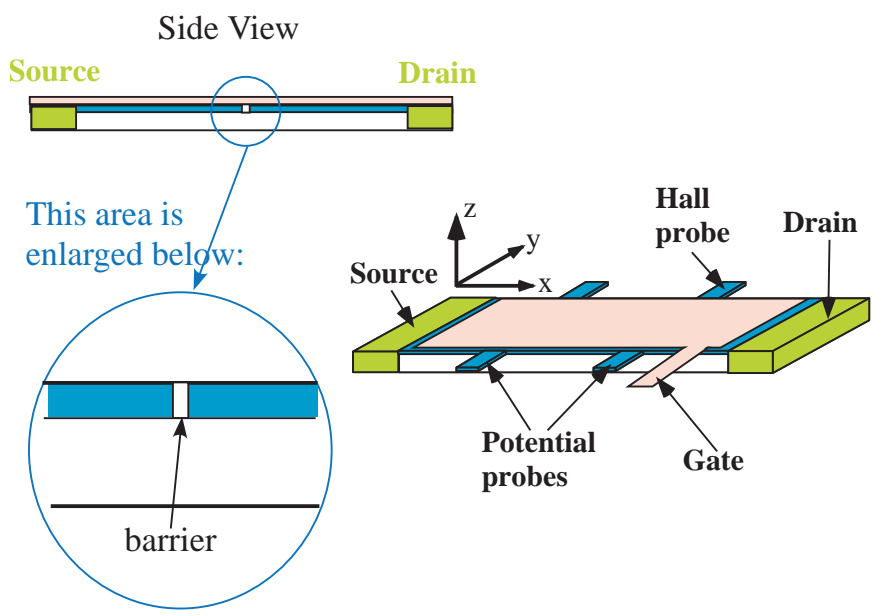

FIG. 1: Type 1: There is a potential barrier near the center of electron channel.

this ultra-thin part from left to right. The length of the ultra-thin part is allowed to be longer than $2 \mathrm{~nm}$. This type of junction is familiar in superconducting phenomena. When the current value exceeds the critical current value of the central thin part, this ultra-thin part plays a role of potential barrier. This type of junction is relatively-easy-to-make compared with the type 1 of quantum Hall device.

We consider a new experiment to observe a tunneling effect in quantum Hall device. Magnetic field is applied as in the direction vertical to the $2 \mathrm{D}$ electron system in a new device as shown in Figs. 1-3. The strength of the magnetic field is adjusted to make an IQH state or an FQH state. Next, an oscillating magnetic field with the frequency value $f$ is added to the constant magnetic field. (We can use an oscillating current adding to the constant current instead of an oscillating magnetic field.) Then we measure the voltage between two potential probes (see Figs. 1-3.), while the electric current value between the source and the drain is changed from small to large. The voltage $V$ versus electric current $I$ are observed for several 

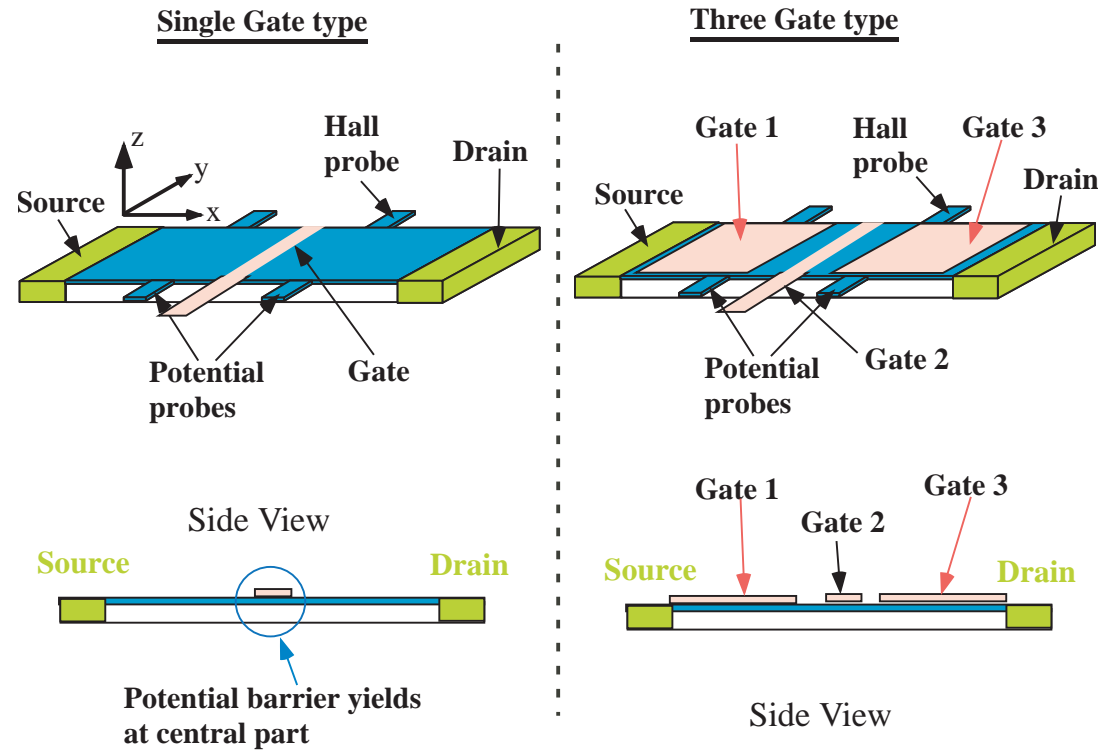

FIG. 2: Single gate type is illustrated in the left hand side. Three gate type is shown in the right hand side.

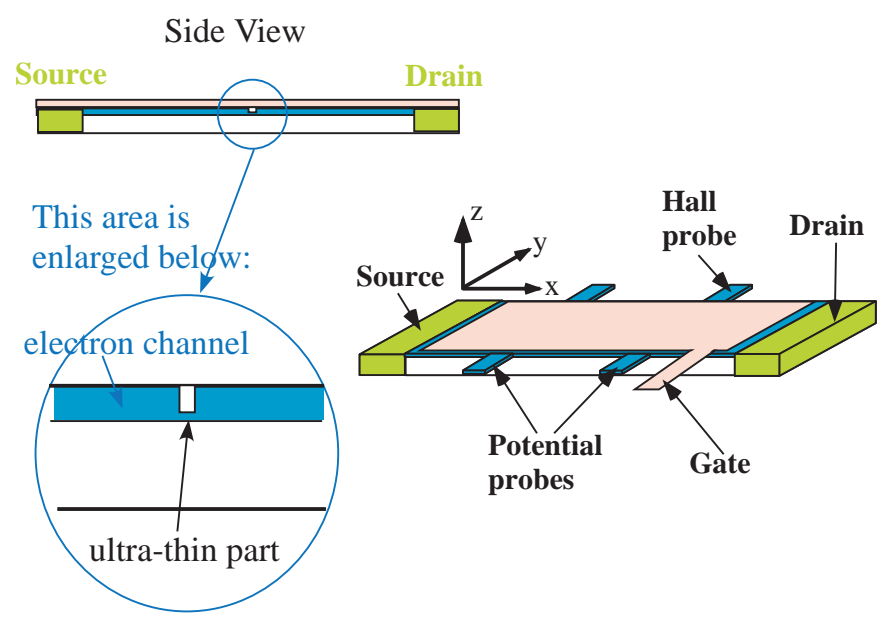

FIG. 3: The central part of electron channel has an ultra-thin thickness as in the enlarged side view.

frequency values of $f$. Observation of $(I, V)$ curve gives us various informations for tunneling effect in quantum Hall states. We examine the properties of the curves in the further sections.

\section{TRANSFER CHARGE THROUGH POTENTIAL BARRIER}

The integer quantum Hall effect is caused by discrete eigenenergy levels (Landau levels) in 2D electron system, where the value of transfer charge across a potential barrier is the elementary charge of electron, $e$.

The confinement of the Hall resistance has been also observed at fractional filling factors $\nu(=2 / 3,1 / 3,1 / 5$ ...) in high mobility semiconductor heterojunctions. The confinement is extremely precise as clarified in the experimental data. However, the confinement at the fractional filling factors $\nu$ cannot be explained by single electron theory. Accordingly the FQHE phenomena are caused by
Potential for electron
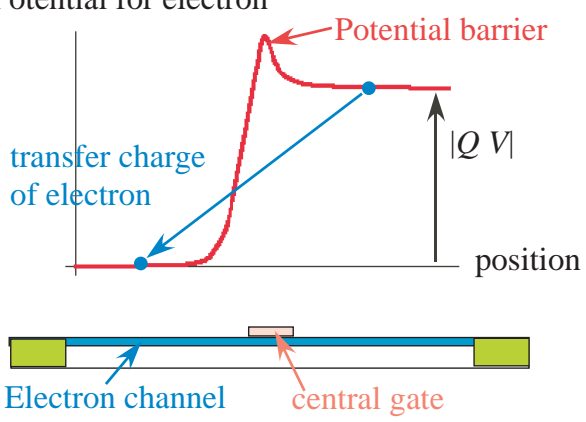

Quantum Hall device

FIG. 4: Position dependence of potential value for quasiparticle with negative charge.

Coulomb interactions among many electrons. Laughlin proposed an explicit trial wave function (Laughlin wave function). He argued that the elementary excitations are quasiparticles with fractional electric charge [4], and explained FQHE. Haldane [5] and Halperin [6] extended the scheme of Laughlin. The quasiparticles and quasiholes have fractional charge and obey fractional statistics [7].

A model of composite fermions has been introduced by Jain [8] and has been developed by many physicists $[9,10]$. The composite fermion consists of an electron (or hole) which is bound to an even number of magnetic flux quanta. Accordingly confinement of Hall resistance is ensured at several fractional filling factors because of binding with magnetic flux quanta. In this model, there are two viewpoints for an transfer charge of a composite fermion.

(The first viewpoint): The transfer charge is $\nu e$ at the filling factor $\nu$.

(The second viewpoint): The transfer charge is $e$ at any filling factor $\nu$. In this case the composite fermions have elementary charge, just like electrons.

Accordingly the transfer charge is different from each other in the two viewpoints. We summarize the trans- 


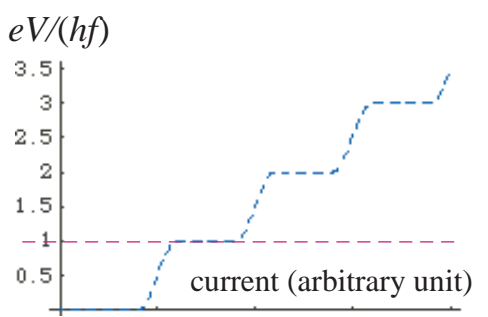

(Case A) Integer quantum Hall states

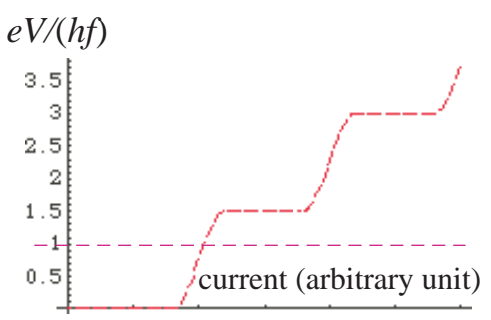

(Case B) Laughlin wave function

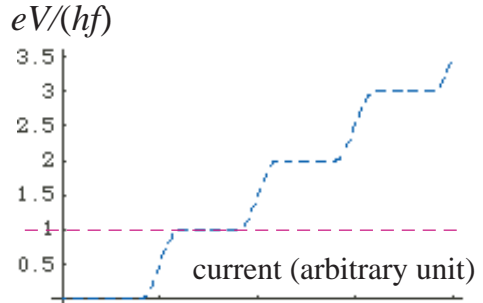

(Case C) for second view point

FIG. 5: Steps of voltage for several theories (Case A indicates the $(I, V)$ curve at IQH state. Case B and Case C are at the filling factor $2 / 3)$.

fer charge value $Q$ across a potential barrier in integer

quantum Hall effect and fractional quantum Hall effect.

Case A: $Q=-e$ for integer quantum Hall states,

Case B: $Q=-\nu e$ for Laughlin theory or for first viewpoint of composite fermion theory,

Case C: $Q=-e$ for second viewpoint of composite fermion theory,

where the value $e$ expresses the elementary charge and $\nu$ indicates the fractional filling factor. If we detect the charge value of $Q$ in the tunneling effect, then the property of FQHE is more clarified.

\section{OBSERVATION OF TUNNELING EFFECT IN QUANTUM HALL DEVICE}

We consider the tunneling effect through the potential barrier in new devices drawn in Figs. 1-3. The potential value of a quasi-particle is shown in Fig. 4.

The electric current flows from the left to the right in Fig. 4. Then the voltage in the left part of the device is higher than one in the right part by $V$, because there is a potential barrier in the central part of the electron channel. The charge of electron is negative value, and therefore the potential value for electron (or a quasi-particle with negative charge) in the right part of the device is higher than in the left part. The behavior of the potential is shown in Fig. 4. Then the quasi-particle with negative charge passes from the right part to the left part through the potential barrier as in Fig. 4.

Magnetic field is applied in the direction vertical to the $2 \mathrm{D}$ electron system. The strength of the magnetic field is adjusted to make an IQH or FQH state. Next, an oscillating magnetic field with the frequency value $f$ is added to the device. (We can use an oscillating current adding to the constant current instead of an oscillating magnetic field.) Then the tunneling effect is stimulated, when the energy difference $|Q V|$ of a quasi-particle is equal to the energy of photon $2 \pi \hbar f$ induced by the magnetic oscillation, where $2 \pi \hbar$ indicates the Planck constant.

We measure the voltage between two potential probes, while the electric current value between the source and the drain is changed from small to large. The graph of voltage versus electric current might have many steps. The predicted behaviors are schematically drawn in Fig. 5. (These phenomena are similar to the ac-Josephson effect, and however the charge values in IQH and FQH states are different from in superconducting phenomena.)

The voltage value at the first step is calculated by the following Eq. (4).

$$
|Q V|=2 \pi \hbar f=h f
$$

where $V$ is the voltage value between two potential probes, and $h$ is the constant of Planck. We should choose the value of $f$ without exceeding the binding energy of the FQH state [11]. For $\nu=2 / 3$ the value of $f$ should be smaller than $4.2 \mathrm{GHz}$ at $B \approx 6.2 \mathrm{~T}$ [11]. Accordingly the value of $f$ is preferable to be smaller than $1 \mathrm{GHz}$. Then, the value $e V /(h f)$ is 1.5 for Laughlin theory or for first viewpoint of composite fermion theory, 1 for second viewpoint of composite fermion theory and 0.5 for electron pair theory [11] in the fractional filling state with $\nu=2 / 3$. In the case of $\nu=2 / 3$ and $f=1 \mathrm{GHz}$, the value of voltage step is $6.203 \mu \mathrm{V}$ for Case $\mathrm{B}, 4.136 \mu \mathrm{V}$ for Case $\mathrm{C}$ in Fig. 5.
[1] D. C. Tsui and A. C. Gossard, Appl. Phys. Lett. 37, 550 (1981); D. C. Tsui, H. L. Stormer, and A. C. Gossard, Phys. Rev. B 25, 1405 (1982); D. C. Tsui, H. L. Stormer, and A. C. Gossard, Phys. Rev. Lett. 48, 1559 (1982).

[2] H. L. Stormer, Nobel lectures, Physics 1996-2000 (World
Scientific), pp 321.

[3] W. Pan, H. L. Stormer, D. C. Tsui, L. N. Pfeiffer, K. W. Baldwin, and K. W. West, Phys. Rev. Lett. 88, 176802 (2002).

[4] R. B. Laughlin, Phys. Rev. B 273383 (1983); R. B. 
Laughlin, Phys. Rev. Lett. 50, 1395 (1983).

[5] F. D. M. Haldane, Phys. Rev. Lett. 51, 605 (1983).

[6] B. I. Halperin, Phys. Rev. Lett. 52, 1583 (1984); S. M. Girvin, Phys. Rev. B 29, 6012 (1984).

[7] S. C. Zhang, T. H. Hansson, S. Kivelson, Phys. Rev. Lett. 62, 980 (1989).

[8] J. K. Jain, Phys. Rev. Lett. 63, 199 (1989); J. K. Jain, Phys. Rev. B 41, 7653 (1990).

[9] R. K. Kamilla, X. G. Wu, and J. K. Jain, Phys. Rev. Lett. 76, 1332 (1996).
[10] B. Blok and X. G. Wen, Phys. Rev. B 42, 8133 (1990).

[11] S. Sasaki, in Surface Science: New Research, C. P. Norris (Ed.) (Nova Science Publishers, 2006), Chap. 4, pp 103161; S. Sasaki, Physica B 281, 838 (2000); S. Sasaki, Proc. 25th Int. Conf. Phys. Semicond. (Springer, 2001), 925; S. Sasaki, Surf. Sci. 532, 567 (2003); S. Sasaki, Surf. Sci. 566, 1040 (2004); S. Sasaki, J. Phys.: Conf. Ser. 100, 042021 (2008). S. Sasaki, J. Phys.: Conf. Ser. 100, 042022 (2008). 